\title{
Planar-Homeotropic Transition in PS-FLC Induced by an Electric Field During Photopolymerization in the Nematic Phase
}

\author{
B. Caillaud \\ L. Dupont \\ J. L. de Bougrenet de la Tocnaye
}

ENST Bretagne, Optics Department, UMR CNRS Foton, Brest, France

\begin{abstract}
We studied a polymer-stabilized ferroelectric liquid crystal presenting a high polymer concentration. These structures exhibit strong interaction between both components of the mixture. This can be demonstrated by applying an electric field during polymerization: it is thus possible to fix both the tilt angle of the director in all mesogenic phases and the layer tilt in the smectic phase.
\end{abstract}

Keywords: homeotropic; polymer network; polymer-stabilized ferroelectric liquid crystal (PSFLC); tilt angle

\section{INTRODUCTION}

Surface-stabilized ferroelectric liquid crystals (SSFLC) exhibit numerous drawbacks: fastidious control of the bulk structure by surface interactions, difficult control of bistable mode, and so on. To improve the characteristics of FLCs [15], another technique has been intensively studied: polymer stabilization of FLCs. Polymer-stabilized ferroelectric liquid crystals (PS-FLC) exhibit several interesting properties of the smectic $\mathrm{C}^{*}$ phase such as grayscale V-shape electro-optic switching [1-3], fast response times, a zigzag-defect-free structure, and good mechanical stability [4]. Classically, polymerization occurs in the smectic A or smectic $\mathrm{C}^{*}$ phase $[5,6]$, with or without applying an electric field $[7,8]$, but in each case, polymerization is initiated when the smectic layer structure is already shaped, which lends to the

Address correspondence to B. Caillaud, ENST Bretagne, Optics Department, UMR 6082 CNRS Foton, CS 83818, 29238 Brest Cedex 3, France. E-mail: bertrand.caillaud@ enst-bretagne.fr 
observation of the polymer-stabilized quasi-bookshelf structure at the end of process. In the case of photo polymerization in the nematic phase with an applied electric field, it is possible to memorize the tilt of the director using the polymer network. The tilted distribution of the director due to the Frederiks transition in the nematic phase is fixed by the polymer network. This memorization effect is expressed in the smectic phase by an apparent tilt of the smectic layers, which is controlled by the electric field amplitude applied during polymerization.

We present experimental results concerning the structures of PS-FLC obtained after the UV polymerization of a monomer (diacrylate)/ ferroelectric liquid-crystalline mixture containing a high concentration of polymer in the nematic phase $(13 \% \mathrm{wt})$ under a sufficiently strong applied electric field. The PS-FLC obtained with a high-density anisotropic polymer network introduces a significant change in both structure and electro-optic effects compared to SSFLC. The polymer network preserves the homogeneous structure of the liquid crystal. Moreover, a hybrid structure between planar and homeotropic can be obtained.

\section{EXPERIMENTAL}

We use a mixture of Felix 015/100 (Clariant) with a high concentration $(13 \% \mathrm{wt})$ of diacrylate mesogenic monomer RM 257 (Merck) to obtain long polymer fibrils and consequently a strong stabilization of the liquid crystal. This concentration corresponds to the limit of the phase separation of the mixture. The phase sequence of the pure liquid crystal is $\mathrm{Cr}-12^{\circ} \mathrm{C}-\mathrm{Sc}-72^{\circ} \mathrm{C}-\mathrm{Sa}-83^{\circ} \mathrm{C}-\mathrm{N}^{*}-86^{\circ} \mathrm{C}-\mathrm{Is}$. A small amount of the the photo initiator Irgacure 651 (Ciba) is added to initiate the polymerization: $0.2 \%$ wt [9]. The experimental cells are composed of two glass plates with indium tin oxyde (ITO) electrodes. In the middle of one of the two electrodes, a wide band $(500 \mu \mathrm{m})$ of ITO is removed by photolithography to obtain two different parts in the same cell: one part that will be photopolymerized with an electric field and the other part that will not. Then a polyimide aligning film is coated onto the two electrodes. The rubbing directions on the two plates are antiparallel. The cells with $2-\mu \mathrm{m}$ spacers are filled by capillary action in the isotropic phase. The mixture is then cooled to the nematic phase to obtain a homogeneous structure without defects. Finally, we apply a variable-strength electric field to one of the two parts of the cell with an Avtech function generator, and at the same time, we start the polymerization with UV light for 15 min with an optical power density of $5 \mathrm{~mW} / \mathrm{cm}^{2}$ at a wavelength of $365 \mathrm{~nm}$ [10]. 


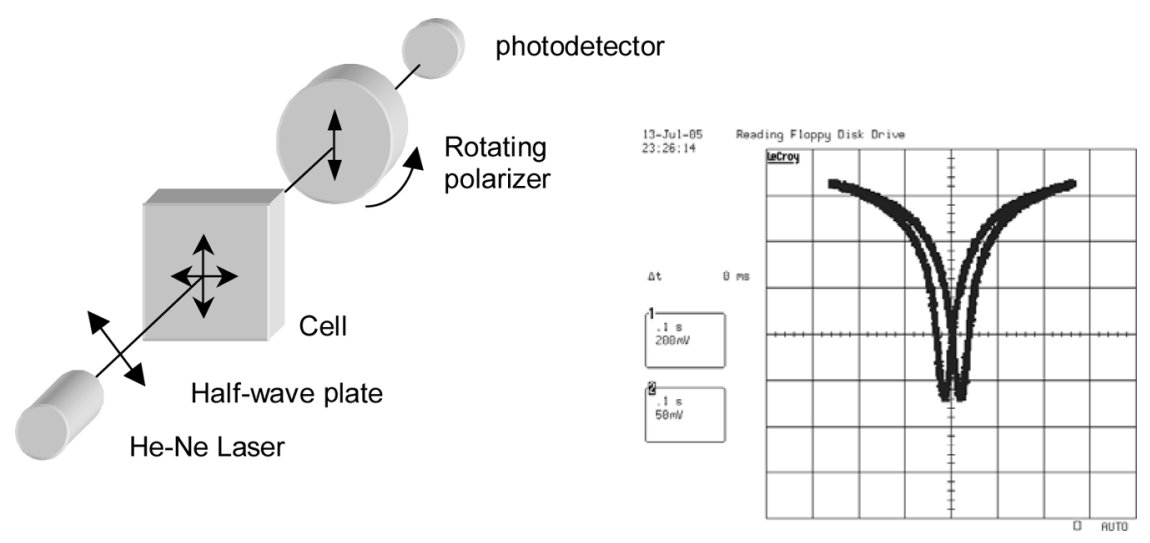

FIGURE 1 Optical setup and V-shape electro-optic response.

When the applied electric field is removed, we observe no change in the nematic phase; the structure seems to be fixed. Finally, the mixture is cooled slowly to room temperature.

The optical characterization setup is made up of a polarized $\mathrm{He}-\mathrm{Ne}$ laser $(632 \mathrm{~nm})$, a half-wave plate to rotate the input polarization, the cell, an analyzer, and a photodiode (Fig. 1). The structures of PS-FLC obtained under polymerization with applied voltage are investigated in different ways.

\section{Qualitative Observations}

The observation of the cells in the $\mathrm{SmC}^{*}$ phase through crossed polarizers gives us information on the structure of the PS-FLC (Fig. 2). First, the director in both areas and for all applied voltages has the same orientation: the rubbing direction (the director orientation coincides with the extinction orientation of the cell). Second, the area with the applied electric field during polymerization exhibits a homeotropiclike structure and a smaller birefringence than the PS-FLC obtained without the applied electric field: it indicates a tilt of the director within the bulk of the cell. Finally, in both cases, the texture of the PS-FLC is homogeneous and smooth without defects (stripes and zigzags).

The cells were observed using scanning electron microscopy (SEM). The cells were frozen in liquid nitrogen and broken (Fig. 3). The liquid crystal was then removed with a specific solvent (isopropanol). In the case of a mixture of diacrylate mesogenic monomer with liquid crystal, the polymerization creates fibrils aligned along the liquid crystal 


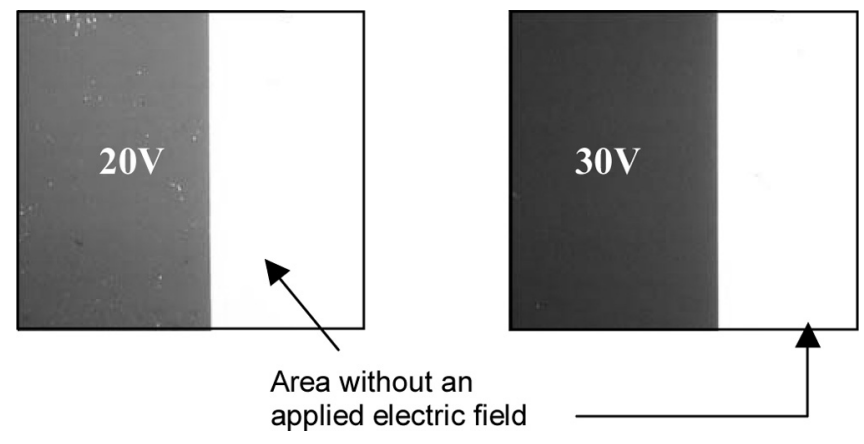

FIGURE 2 Polarizing microscopy images of the cells observed at room temperature. Dark areas are made up of polymer network liquid crystals obtained with voltage applied during polymerization. Bright areas correspond to liquid crystals in a planar geometry.

director [11]. For the cell polymerized without an electric field, the polymer network exhibits uniform polymer fibrils along the length of the substrate, which indicates that the polymer fibrils are oriented along the rubbing direction and parallel to the substrate within the bulk of PS-FLC.

When an electric field is applied during polymerization, the polymer network exhibits an undulating morphology. These structures tend to

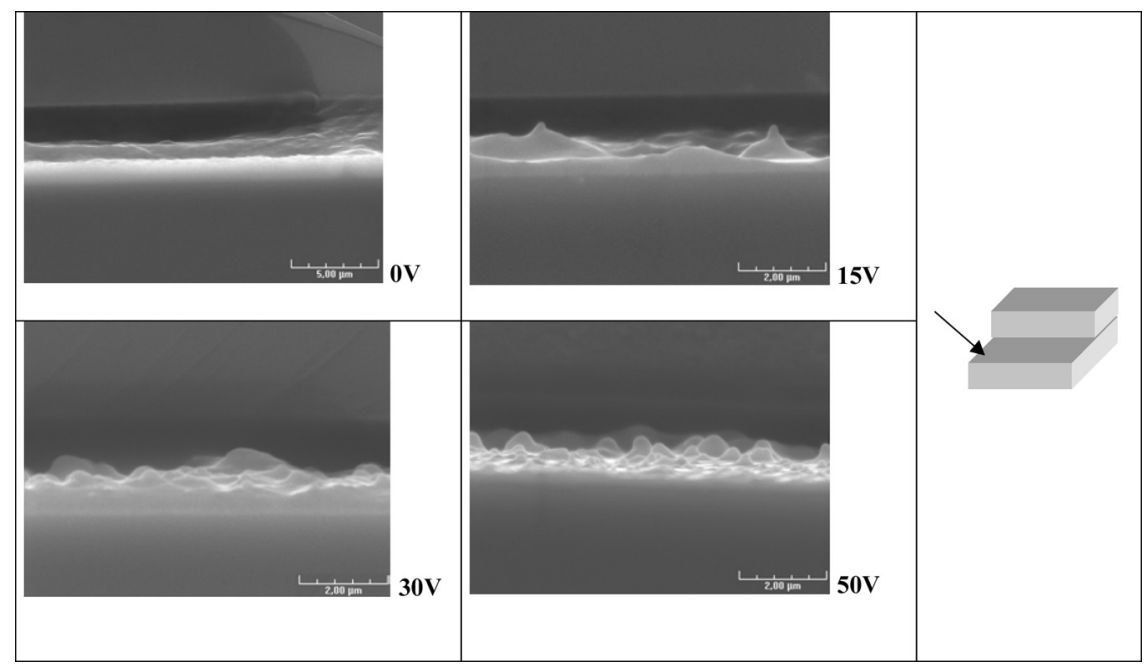

FIGURE 3 Cross-section SEM images of polymer network for different applied voltages $(0,15,30,50 \mathrm{~V})$ during polymerization. 
indicate that the polymer fibrils are not in the plane of the substrate but out of the plane. However, no fibrils connecting both plates are observed in the cross section, only aggregates that seem to be made up of small collapsed polymer fibrils. It is obviously impossible to draw conclusions about the precise orientation of the polymer fibrils, but the evolution of the morphology of the different samples points to a clear difference in structure that depends on the polymerization process.

\section{Optical Measurements}

\section{Electro-optic Response}

We characterized the electro-optic response of the planar PS-FLC. With such a high polymer concentration, the observed electro-optic effect clearly has the grayscale $\mathrm{V}$-shape response with a residual hysteresis. The electro-optic measurements were carried out at $1 \mathrm{~Hz}$ with crossed polarizers.

\section{Contrast Measurement}

Using the optical setup described previously, we measured the contrast of the different cells by increasing the applied voltage (Fig. 4). In the case when an electric field was applied during polymerization, a significant decrease in the contrast can be observed with increasing electric field. These results are in agreement with a progressive tilt of both the director and smectic layers.

\section{Anisotropy and Phase-Shift Measurement}

The phase shift is measured with the optical setup described in Fig. 3 . The input polarization is set at $45^{\circ}$ to the eigenaxes of the liquid

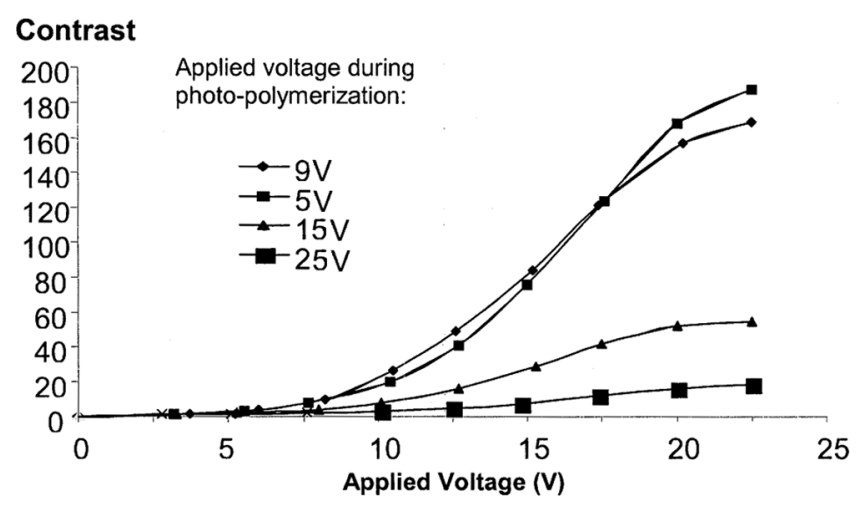

FIGURE 4 Contrast measurement. 
crystal, and the phase shifts of the PS-FLC with and without applied electric field during polymerization are measured at the same time to guarantee the same thickness for both kinds of PS-FLC cell. The light intensity measured through crossed polarizers is $I_{\perp}=I_{0} \sin ^{2}(\Delta \phi / 2)$, and the light intensity measured through parallel polarizers is then $I_{\|}=I_{0}\left(1-\sin ^{2}(\Delta \phi / 2)\right)$. Combining these two relations, the phase shift is easily obtained:

$$
\Delta \phi=2 \sin ^{-1} \sqrt{\frac{I_{\perp}}{I_{\perp}+I_{\|}}} .
$$

The ratio between effective birefringences of the different areas of the same cell can be obtained from the ratio between phase shifts. Considering that in the cell without applied electric-field polymerization, the optical axis is parallel to the substrate, and that with the electric field, the optical axis (and smectic layers) is tilted, it is possible to evaluate the effective out-of-plane tilt angle $\Theta$ of the director using the relation

$$
\frac{1}{n^{2}(\Theta)}=\frac{\cos ^{2} \Theta}{n_{e}^{2}}+\frac{\sin ^{2} \Theta}{n_{o}^{2}} .
$$

The effective birefringence for a tilted liquid crystal is given by the relation

$$
\Delta n_{\text {eff }}=n(\Theta)-n_{0} .
$$

In the case of planar PS-FLCs, the birefringence is given as $\Delta n=n_{e}-n_{o}$. This value takes into account both polymer and liquidcrystal birefringences. The ratio of the phase shifts in each area is then equal to the ratio of birefringences: $\Delta \phi_{V} / \Delta \phi_{0}=\Delta n_{\text {eff }} / \Delta n$. By plotting this ratio as function of the tilt of the director and comparing to the experimental values, the effective tilt angle can be evaluated (Fig. 5).

The measured phase shifts for the cell polymerized without an applied electric field are about $140^{\circ}$ in the smectic and nematic phases and decrease to $20^{\circ}$ in the isotropic phase. For the cells polymerized under an applied electric field, the phase shift is about $20^{\circ}$. In each case, the effective tilt angle does not exhibit an abrupt change in all liquid-crystalline phases (Fig. 6).

Calculating the ratio for each cell, we obtain the ratio of the birefringence and then the effective angle. The measurements of the effective angle $\Theta$ were done in the smectic $\mathrm{C}^{*}$ and isotropic phases and for different applied voltages during polymerization (Fig. 7). An increase of this effective tilt angle of the director is observed in 


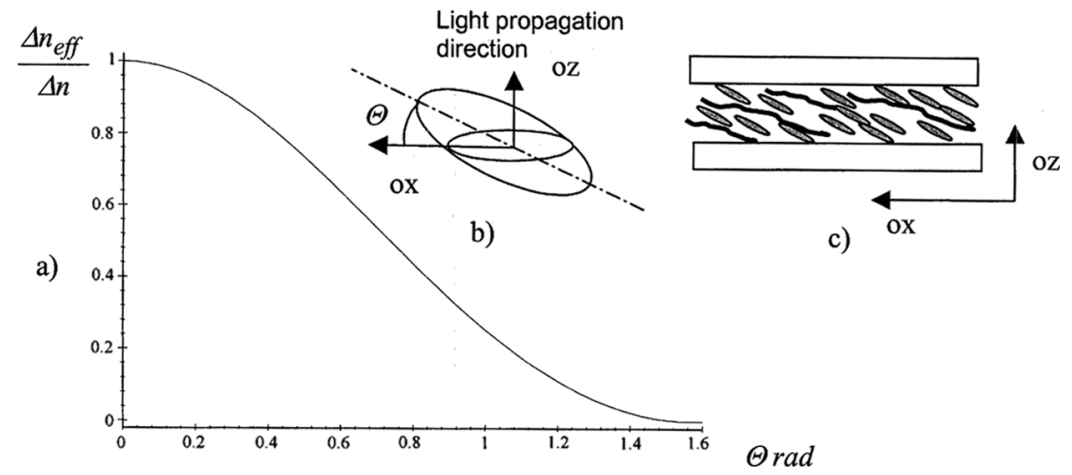

FIGURE 5 a) Plot of the ratio $\Delta n_{\text {eff }} / \Delta n$ versus the out-of-plane tilt angle $\Theta$; b) tilted indices ellipsoid; and c) structure of the director with tilted polymer network.

both the smectic $\mathrm{C}^{*}$ and isotropic phases. However, the measured tilt angle in the isotropic phase (which is the effective angle of the polymer network) is lower than in the smectic $\mathrm{C}^{*}$ phase. Two interpretations can be considered: the director has a greater tilt than the average direction of polymer network in smectic $\mathrm{C}^{*}$, or the intrinsic birefringence of the polymer network is higher when polymerized under an applied electric field than without an electric field.

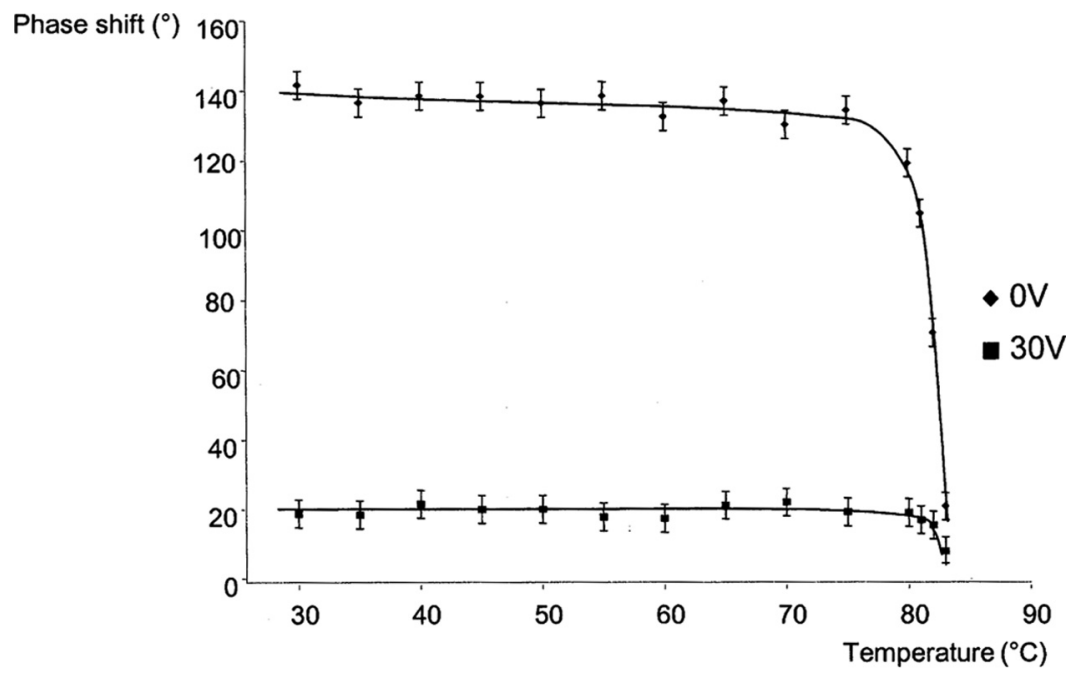

FIGURE 6 Phase-shift evolution for PS-FLC obtained for polymerization with and without an electric field. 


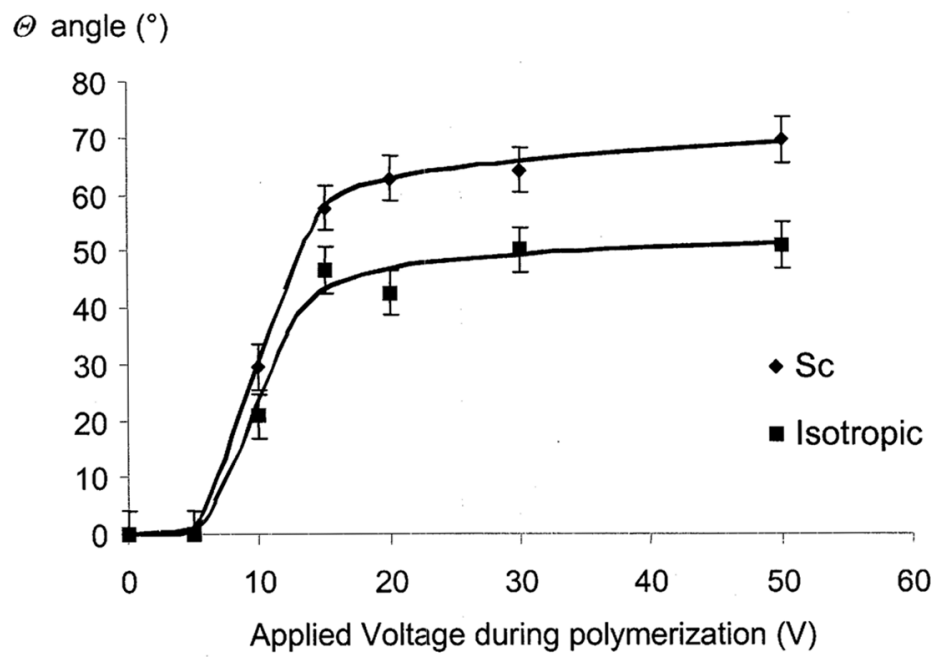

FIGURE 7 Out-of-plane tilt of the director versus the voltage applied during polymerization in the smectic $\mathrm{C}^{*}$ and isotropic phases. In the isotropic phase, the measured birefringence is a result of the anisotropic polymer network.

\section{DISCUSSION}

The polymer network obtained in the nematic phase, with an applied electric field and high concentration of monomer, fixes the reorientation of the director. Both optical observations and measurements show that the out-of-plane tilt angle $\Theta$ of the director remains constant in all mesophases, which clearly results from a strong interaction between the liquid crystal and the polymer network. When the orientation of the director is fixed in the nematic phase and the applied electric field is removed, no change in the structure of the PS-FLC is observed. The measurement of anisotropy versus temperature of the area with a tilted optical axis shows this constrained structure is stable in all mesogenic phases.

The SEM images do not allow us to fully understand these interactions because the polymer network is only observable on the substrate surfaces and not between the substrates. We observe significant differences in the polymer network morphology for the different kinds of polymerization, but, for instance, it is not possible to clearly observe the fibrils and thus to establish a relation between the measured tilt angle of the liquid crystal and the tilt angle of polymer network. Further experiments with SEM observations are necessary to determine the morphology of the network more precisely. 
Observations with a polarizing microscope show that no stripe and zigzag defects are present, which tends to indicate that the structure of the smectic layers in the smectic $\mathrm{C}^{*}$ phase is not comparable to that in SSFLC. With our optical experiments, we are able to measure an average out-of-plane director orientation within the bulk; however, two unknown parameters remain: the director distribution and the structure of smectic layers [12]. With such a high polymer concentration, the layer geometry cannot be deduced from the SSFLC structure and described precisely. To overcome these difficulties, studies with X-ray diffraction, for example, could give precious new information about these structures [13,15]: Whether they have a chevron structure or tilt of the layers, etc.

Studies on smectic layer structures of PS-FLC with nonmesogenic monomers [14] have already shown that the chevron-like structure of smectic layers strongly depends on the UV light intensity but only weakly on polymer concentration. However, with mesogenic polymers, the interactions between the polymer network and the liquid crystal are stronger, and the impact of monomer concentration on the smectic layers can be greater and the structure significantly different.

\section{CONCLUSION}

The polymer network obtained with high concentrations of monomer exhibits several interesting properties: as with the classical polymer network, the V-shaped contrast curve is obtained with standard applied voltages. The network suppresses all defects related to the ferroelectric phase, giving an excellent optical extinction level for relatively low switching voltages with good mechanical strength. In addition, the application of electric fields during plymerization enables good control of the out-of-plane tilt of the director. This property can be very useful when precisely adjusting the effective birefringence of the nematic or smectic liquid-crystalline material and when freezing it after polymerization. For example, with the same liquid crystal in a nematic phase, it is possible to generate and memorize different phase shifts at zero field. This process enables the design of pixelled optical components such as polarization-sensitive holograms that can be turned off by the application of an electric field.

\section{REFERENCES}

[1] Furue, H., Yokoyama, H., \& Kobayashi, S. (2001). Jap. J. Appl. Phys., 40(9B), 5790-5794.

[2] Li, J., Zhao, W., Ma, K., \& Huang, X. (1998). Mol. Liq. Crys., 321, 395-402. 
[3] Kobayashi, S., Xu, J., Furuta, H., Murakami, S., Kawamoto, S., \& Oh-kouchi, M. (2004). Opt. Eng., 43(2), 290-298.

[4] Fujikake, H., Murashige, T., Sato, H., Iineo, Y., Kawakita, M., \& Kikuchi, H. (2002). J. Soc. Information Display, 10(1), 95-99.

[5] Furue, H., Iimura, Y., Hasebe, H., Takatsu, H., \& Kobayashi, S. (1998). Mol. Crys. Liq. Cryst., 317, 259-271.

[6] Lee, S. N. \& Chien, L. C. (2004). Mol. Crys. Liq. Cryst., 410, 183-190.

[7] Li, J., Zhu, X., Xuan, L., \& Huang, X. (2002). Ferroelectric, 277, 85-105.

[8] Murashige, T., Fujikake, H., Ikehata, S., \& Sato, F. (2004). Elect. Commun. Jpn. Part 2, 87(4), 16-24.

[9] Guymon, C. A., Hoggan, E. N., Walba, D. M., Clark, N. A., \& Bowman, C. N. (1995). Liq. Cryst., 19(6), 719-727.

[10] Nourry, J., Vigouroux, A., Maganldo, A., Sixou, P., Mitov, M., Boudet, A., Glogarova, M., \& Bubnoy, A. M. (1998). Ferroelectric, 212, 203-210.

[11] Li, J., Wang, Z., Cai, Y., \& Huang, X. (1998). Ferroelectric, 213, 91-99.

[12] Pirs, J., Bos, P., Petrovsek, R., Kralj, S., Pirs, S., Zumer, S., \& Matuszczyk, T. (2002). Jpn. J. Appl. Phys., 41, 6011-6015.

[13] Fujikake, H., Toyooka, T., Murashige, T., Sato, H., Iino, Y., Kawakita, M., \& Kikuchi, H. (2002). Liq. Cryst., 29, 881-887.

[14] Fujikake, H., Aida, T., Takizawa, K., Kikuchi, H., Fujii, T., \& Kawakita, M. (1999). Elect. Commun. Jpn. Part 2, 82(8), 1-8.

[15] Hikmet, R. A. M., Boots, H. M. J., \& Michielsen, M. (1995). Liquid Crystals, 19, $65-76$. 